\title{
Isolation, identification, and anti-inflammatory activity of polysaccharides of Typha angustifolia
}

Huan Wei ${ }^{\mathrm{a}, 1}$, Yuqi Shi ${ }^{\mathrm{b}, 1}$, Zhixiang Yuan ${ }^{\mathrm{b}, 1}$, Zhinan Huang ${ }^{\mathrm{a}}$, Fuhong Cai ${ }^{\mathrm{a}}$, Jingfeng Zhu ${ }^{\mathrm{a}}$, Wanwan Zhanga ${ }^{\mathrm{a}}$, Jia Lia ${ }^{\mathrm{a}}$, Qingping Xiong ${ }^{\mathrm{a}}$, Yunpeng Wang ${ }^{\mathrm{a}}$, Xiaoli Wang,

a Jiangsu Provincial Key Construction Laboratory of Probiotics Preparation, College of Life Science and Food Engineering, Huaiyin Institute of Technology, Huai'an 223003, China

${ }^{b}$ College of Plant Protection, Nanjing Agricultural University, Nanjing 210095, China

${ }^{1}$ Huan Wei, Yuqi Shi and Zhixiang Yuan contributed equally to this study and are regarded as joint first authors

* Corresponding author: College of Life Science and Food Engineering, Huaiyin Institute of Technology, Huai'an 223003, China; Tel.: +86-517-83559216, fax: +86517-83559216, e-mail: xlwang@hyit.edu.cn 


\section{Nuclear magnetic resonance spectroscopy (NMR)}

PTA-2(30mg) was completely dissolved in deuteroxide $\left(\mathrm{D}_{2} \mathrm{O}\right)$, and then freezedried to obtain the powder. The above operation was repeated six times and the sample was dissolved in $\mathrm{D}_{2} \mathrm{O}(0.5 \mathrm{~mL})$ again. ${ }^{1} \mathrm{H}$ NMR and ${ }^{13} \mathrm{C}$ NMR spectrum were recorded by the JNM-ECZR NMR spectrometer.

In most situations, the ${ }^{1} \mathrm{H}$ NMR and ${ }^{13} \mathrm{C}$ NMR spectrum were used to analyze the configuration of the glycosidic linkages. The anomeric regions of $\alpha$-configuration appear in 5.0-5.8 ppm (anomeric proton) and 98-103 ppm (anomeric carbon), while $\beta$ configuration relatively corresponds to $4.4-5.0 \mathrm{ppm}$ and $103-106 \mathrm{ppm}$ in anomeric regions. As shown in Figure S1 and Figure S2, the useful anomeric signals were 85.09, $\delta 5.03, \delta 4.94, \delta 4.78, \delta 4.58$ and $\delta 4.54\left({ }^{1} \mathrm{H}\right.$ NMR spectrum), the ones in ${ }^{13} \mathrm{C}$ NMR spectrum were $\delta 104.02, \delta 103.86, \delta 103.55, \delta 103.02, \delta 101.09$ and $\delta 99.35$. Combined with IR Spectra, it could be inferred that the main chains of PTA-2 consist of $\beta$ glucosidic bonds, as well as a few side chains modified by $\alpha$ glucosidic bonds. 

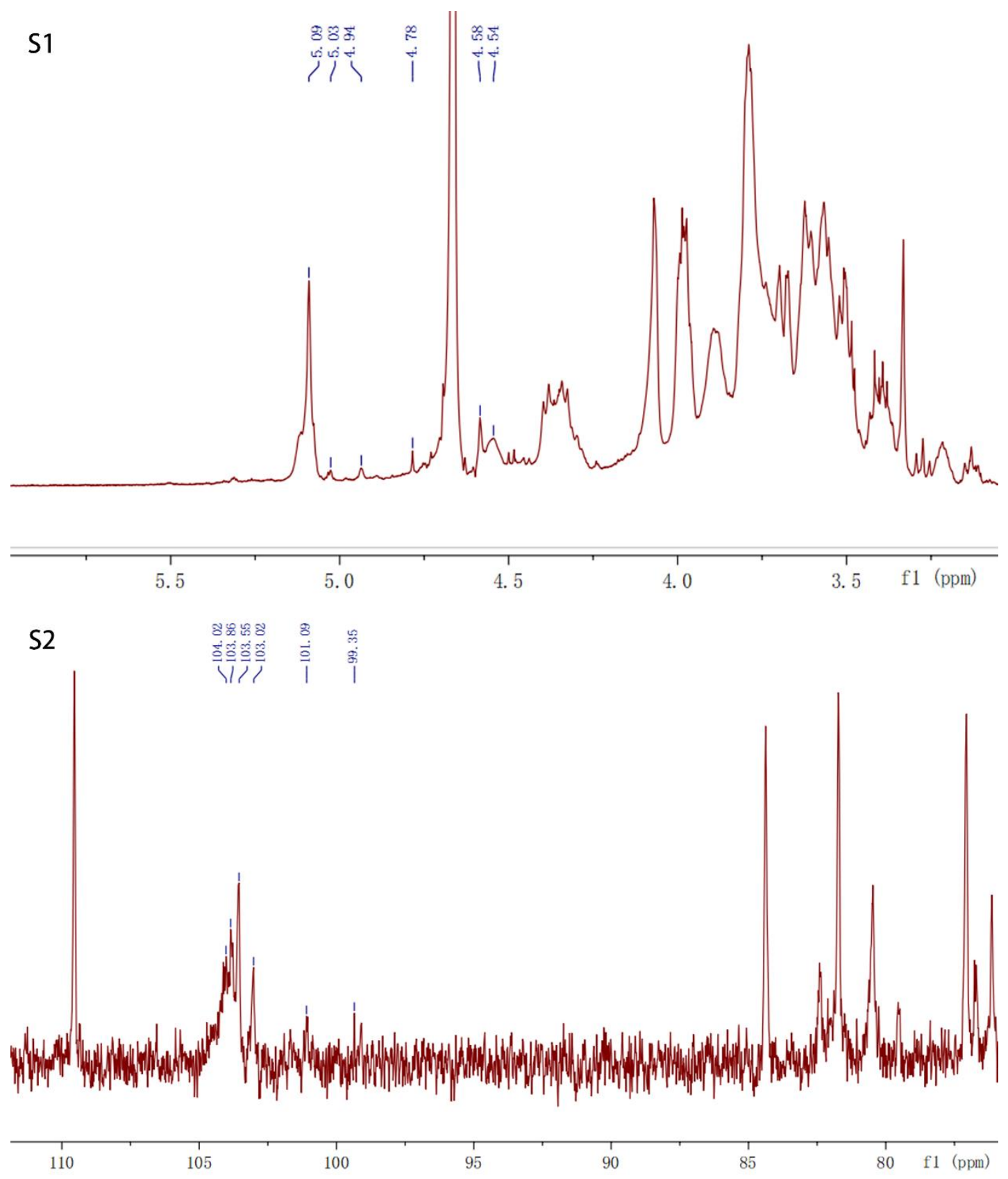

Fig. S. The ${ }^{1} \mathrm{H}$ NMR spectrum of PTA-2 (S1) and ${ }^{13} \mathrm{C}$ NMR spectrum of PTA-2 (S2). 